Supporting Information

\title{
Separation of a Close-Boiling 1,2-Propanediol and Ethylene Glycol Mixture Using Pressure-Related Distillation
}

Cong Duan, Chunli Li*, Hao Li

National-Local Joint Engineering Laboratory for Energy Conservation in Chemical Process Integration and Resources Utilization, School of Chemical Engineering and Technology, Hebei University of Technology, Tianjin, China

Table S1. Economic basis ${ }^{1}$

\begin{tabular}{|c|c|}
\hline parameter & value \\
\hline \multicolumn{2}{|l|}{ Condensers \& Preheaters } \\
\hline Heat transfer coefficient & $0.852 \mathrm{~kW} / \mathrm{K} \cdot \mathrm{m}^{2}$ \\
\hline Capital cost (area in $m^{2}$ ) & $7296(\text { area })^{0.65}$ \\
\hline \multicolumn{2}{|l|}{ Reboiler } \\
\hline Heat transfer coefficient & $0.568 \mathrm{~kW} / \mathrm{K} \cdot \mathrm{m}^{2}$ \\
\hline Capital cost (area in $m^{2}$ ) & $7296(\text { area })^{0.65}$ \\
\hline \multicolumn{2}{|l|}{ Columns } \\
\hline Column diameter ( $\mathrm{D}$ in meters) & Aspen tray sizing \\
\hline Column length ( $\mathrm{L}$ in meters) & $\mathrm{L}=1.2 \times 0.61 \times\left(\mathrm{N}_{T}-2\right)$ \\
\hline Column vessel capital cost & $17640 \mathrm{D}^{1.066} L^{0.802}$ \\
\hline Compressor $^{2}$ & $(1625.9 / 280) \times 517.5 \times B H P^{0.82} \times(2.11+1)$ \\
\hline \multicolumn{2}{|l|}{ Energy cost } \\
\hline HP steam & $\$ 9.88 / \mathrm{GJ}\left(41 \mathrm{barg}, 254^{\circ} \mathrm{C}\right)$ \\
\hline MP steam & $\$ 8.22 / \mathrm{GJ}\left(10 \mathrm{barg}, 184^{\circ} \mathrm{C}\right)$ \\
\hline Cooling water & $\$ 0.354 / \mathrm{GJ}$ \\
\hline Electricity & $\$ 16.8 / \mathrm{GJ}$ \\
\hline TAC & (Capital cost/payback period) + energy cost \\
\hline Payback period & 3 years \\
\hline
\end{tabular}




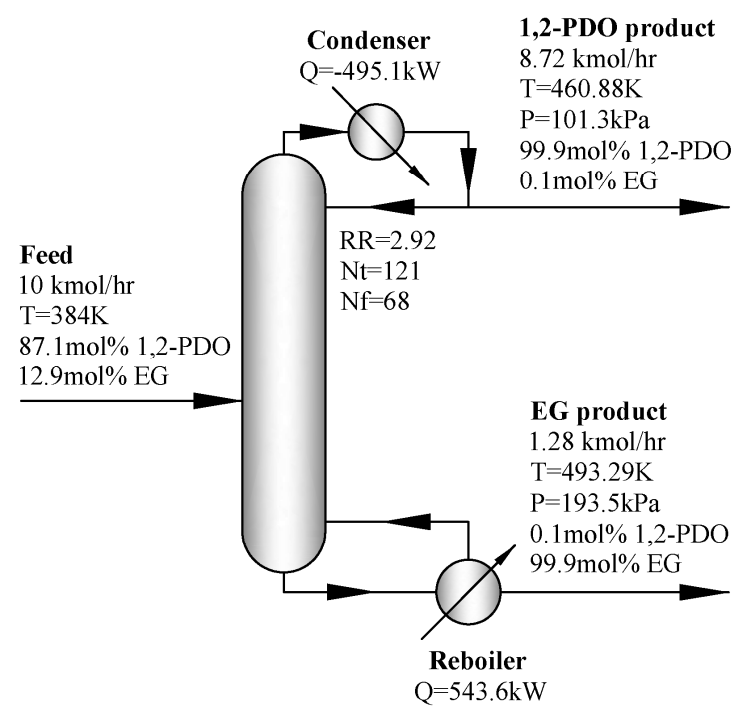

Figure S1. An optimal single-column distillation process at $101.3 \mathrm{kPa}$ proposed by Chen et al. ${ }^{3}$

\section{- REFERENCES}

(1) Luyben, W. L. Comparison of extractive distillation and pressure-swing distillation for acetone/chloroform separation. Comput. Chem. Eng. 2013, 50, 1.

(2) Kiran, B.; Jana, A. K. Assessing the performance improvement of an intensified heat integration scheme: Reactive pressure-swing distillation. Appl. Therm. Eng. 2015, 76, 509.

(3) Chen, Y. C.; Hung, S. K.; Lee, H. Y.; Chien, I. L. Energy-saving designs for separation of a close-boiling 1,2-propanediol and ethylene glycol mixture. Ind. Eng. Chem. Res. 2015, 54 (15), 3828. 